\title{
Um estudo de técnicas aplicadas ao reconhecimento da língua de sinais: novas possibilidades de inclusão digital
}

\author{
Evandro Metz Flores, Jorge Luis Victória Barbosa, Sandro José Rigo \\ UNISINOS - Universidade do Vale do Rio dos Sinos \\ evandrometzflores@gmail.com,jbarbosa@unisinos.br, rigo@unisinos.br
}

\begin{abstract}
The inclusion of the deaf in the hearing society collides with the listener's lack of knowledge about the Brazilian Sign Language (LIBRAS). Some studies aim to overcome this deficiency with the development of automated tools of support. Although some prior work results can be considered inappropriate, the event of some new technologies can indicate good results. This work is a survey of research approaches for detection and translation of sign language. The current possibilities in this area are analyzed, along with the description of necessities for a functional interpretation.
\end{abstract}

Keywords: Deaf, Sign language, Automated tool of support

Resumo. A inclusão do surdo na sociedade ouvinte esbarra na falta de conhecimento do ouvinte sobre a Lingua Brasileira de Sinais (LIBRAS). Alguns trabalhos visam sanar esta deficiência com o desenvolvimento de ferramentas automáticas de apoio. Apesar de algumas iniciativas não terem apresentado resultados adequados, novas tecnologias permitem identificar possibilidades promissoras. Neste trabalho é apresentado um levantamento de pesquisas para a detecção e tradução da língua de sinais, complementado com a análise de possibilidades atuais e com a descrição de necessidades para uma interpretação funcional da LIBRAS.

Palavras-Chaves: Surdo, Língua de Sinais, Ferramentas Automáticas de suporte

\section{Introdução}

No Brasil, a educação de surdos vem demandando esforços na qualificação e formação de pessoal, para atender ao que está oficializado na Língua Brasileira de Sinais (LIBRAS), com base na Lei N. ${ }^{\circ} 10.436$ de 24 de abril de 2002, posteriormente regulamentada pelo decreto $\mathrm{N}^{\circ}$ 5.626, de 22 de dezembro de 2005. Esta legislação fortalece o enfoque bilíngue, a partir do qual se considera que a primeira língua do indivíduo surdo deve ser a Língua de Sinais, seguida pela língua portuguesa na sua modalidade escrita. Já a modalidade oral da língua portuguesa pode também ser adotada, mas como uma escolha pessoal destes sujeitos.

A partir destes pressupostos, observa-se um movimento de inclusão escolar que já resulta em um grande número de alunos surdos frequentando as escolas regulares em diferentes níveis de ensino. Ao mesmo tempo destacam-se movimentos de capacitação de professores, para que incorporem habilidades de uso da Língua de Sinais. Complementa este contexto a presença de interpretes e tradutores que realizam atividades de apoio para a comunicação dos surdos em ambiente escolar onde o professor não domina a Língua de Sinais. Entretanto, de forma geral, constata-se a 
necessidade de avanços nos diversos processos de capacitação e de apoio em LIBRAS [Leite, 2007].

São conhecidas abordagens de uso de tecnologias para apoio na formação dos professores [Metz, 2011], bem como são relatados trabalhos que visam sanar esta deficiência com o desenvolvimento de ferramentas automáticas de apoio, desenvolvidas no contexto de aplicação de recursos de Informática na Educação, explorando possibilidades de recursos tecnológicos [Lira, 2003]. Apesar de algumas iniciativas não terem apresentado resultados considerados adequados ou suficientes, novas tecnologias permitem identificar possibilidades promissoras. Neste trabalho é apresentado um levantamento de pesquisas para a detecção e tradução da língua de sinais, sendo complementado com a análise de possibilidades atuais e com a descrição de necessidades para uma interpretação funcional da LIBRAS. Estes trabalhos avançam em relação a resultados anteriores, incorporando recursos importantes que permitem explorar a detecção automática de sinais [Tavares et al, 2010][Yang, 2010] [Kin et al, 2012]. Os resultados desta detecção automática podem ser disponibilizados com uso de tecnologias bem estabelecidas promovendo a interação multimodal, tais como as tecnologias de reconhecimento e síntese de voz.

Este estudo apresenta aspectos relevantes para o contexto educacional, pois o avanço de recursos tecnológicos, tal como será descrito, possibilita a implementação de soluções viáveis para o apoio nas atividades de tradução e detecção de mensagens na Língua de Sinais. Bons resultados nestas iniciativas podem significar a disponibilização, em larga escala, de dispositivos de apoio para o indivíduo surdo, permitindo a interpretação e tradução funcional de mensagens em LIBRAS. Este resultado irá ampliar as suas possibilidades de interação direta com os grupos de colegas em contexto escolar e ao mesmo tempo diminuirá a dependência que se observa em relação ao tradutor ou ao professor habilitado em LIBRAS. Apesar de não haver ainda uma solução definitiva para esta questão, são considerados como exemplos que demonstram claramente avanços significativos nesta questão: a) os resultados apresentados até o momento; b) a ampliação do número de iniciativas de pesquisa; c) a exploração de diferentes dispositivos digitais.

\section{A língua de Sinais}

Uma das crenças mais recorrentes quando se fala na língua de sinais é que ela é universal. Porém o que se pode dizer universal é o impulso dos indivíduos para a comunicação e, no caso dos surdos, esse impulso é sinalizado. A língua dos surdos não pode ser considerada universal, dado que não pode ser utilizada por todos os surdos de todas as sociedades de maneira uniforme e sem influência de uso. $\mathrm{Na}$ busca da universalidade, está também implícita uma tendência a simplificar a riqueza linguística, sugerindo que talvez para os surdos fosse mais fácil se todos usassem uma língua única, uniforme.

A língua de sinais dos surdos é natural, pois evoluiu como parte de um grupo cultural do povo surdo. Em meados da década de 1970, o comitê da Comissão da Unificação de Sinais propôs um sistema padronizado de sinais internacionais, o "gestuno", tendo como critério a seleção de sinais mais compreensíveis, que facilitassem o aprendizado, a partir da integração das diversas línguas de sinais. A comunidade surda, de forma geral, não considera o gestuno uma língua real, uma vez que foi inventada e adaptada. O nome gestuno é de origem italiana e significa "unidade em língua de sinais" [GESSER, A. 2009]. 
Ao descrever os níveis fonológicos e morfológicos da língua americana de sinais, William Stokoe apontou três parâmetros que constituem os sinais e nomeou-os: configuração de mão (CM); ponto de articulação (PA) ou locação (L), delimitado na figura por uma elipse; e movimento (M), cuja a direção indicada por uma seta, como pode ser visto na (Figura 1).

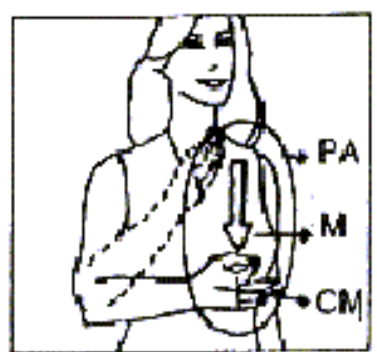

Figura 1 - Níveis fonológicos e morfológicos segundo William Stokoe [Brito 2011].

A partir da década de 1970, os linguistas Robbin Battison (1974), Edward S. Klima \& Ursulla Bellugi conduziram estudos aprofundados sobre a gramática da língua americana de sinais, especificamente sobre os aspectos fonológicos, descrevendo um quarto parâmetro: a orientação da palma da mão $(\mathrm{O})$. Ficou demonstrado que dois sinais com os mesmos outros três parâmetros iguais $(\mathrm{CM}, \mathrm{L}, \mathrm{M})$ poderiam mudar de significado de acordo com a orientação da mão [GESSER, A. 2009].

Além das mãos existem outros recursos para produzir informações linguísticas. Existem também os marcadores não manuais que são extremamente utilizados pelos surdos. Para compor a estrutura da língua são utilizadas as expressões faciais (movimento de olhos, boca, cabeça, sobrancelha e etc.) como elementos gramaticais.

Ainda muito forte, a crença da sociedade ouvinte de que a língua de sinais não tem gramática, baseia-se no fato de que ela seria composta apenas por mímicas e pantomimas. Porém a pantomima quer fazer com que o interlocutor veja o objeto representado, enquanto o sinal quer destacar o símbolo convencionado para tal. É possível expressar conceitos abstratos na língua de sinais. Assim como os falantes de línguas orais, os falantes de língua de sinais podem, por exemplo, discutir filosofia, política, elaborar poesias, peças teatrais, contar piadas, contar e inventar histórias.

O fato da língua de sinais ser uma língua de modalidade espaço-visual faz com que haja uma tendência em se pensar que é uma língua exclusivamente icônica. Embora existam muitos sinais icônicos, essa característica se faz presente também nas línguas orais, onde também é incorporada essa característica. Por exemplo, no caso das onomatopéias como "pingue-pongue", "zum-zum" ou "tique-taque".

Mesmo sinais icônicos tendem a variar de uma língua de sinais para outra. O alfabeto manual, utilizado para soletrar manualmente as palavras (datilologia ou soletramento digital), é apenas um recurso utilizado por falantes da língua de sinais. Não é uma língua e não representa a língua de sinais como um todo, mas sim um código de representação das letras alfabéticas. A Figura $2^{\mathrm{a}}$, a seguir, representa o alfabeto manual da LIBRAS.

O alfabeto manual brasileiro é composto por 27 formatos, contando o grafema "ç", que é a configuração de mão da letra "c" com movimento trêmulo. O alfabeto manual varia para cada país. $\mathrm{O}$ alfabeto britânico, que pode ser visto na Figura $2 b$, por exemplo, é representado com o uso das duas mãos. 


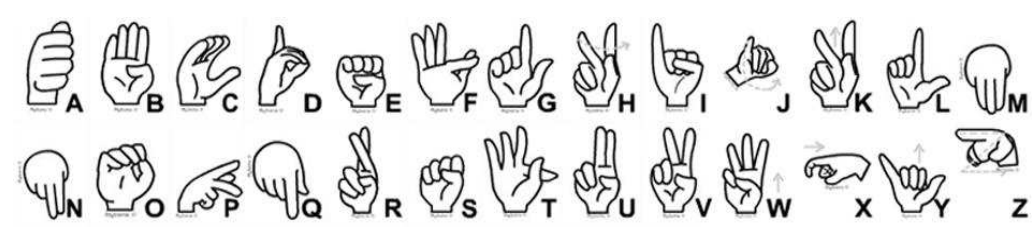

(a)

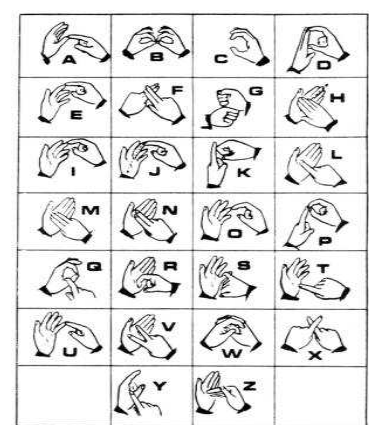

(b)

Figura 2 - Alfabetos Manuais. a: LIBRAS [Acessibilidade Brasil 2011].

b: Britânico [Inforgestual 2011].

A partir da contextualização de elementos da Língua de Sinais, a seguir serão analisados trabalhos com o intuito de realização de sua tradução automática.

\section{Trabalhos Pesquisados}

A busca por recursos de apoio às necessidades de interação envolvendo os indivíduos surdos pode ser observada em trabalhos diversos, inicialmente com uso prioritário de recursos de Computação Gráfica e Animação, juntamente com recursos de Síntese e Reconhecimento de Voz [Coradine, 2002; LIRA, 2003]. Avaliações realizadas acerca da viabilidade e alcance destes trabalhos iniciais apontavam para dificuldades em abrangência de sinais detectados e para restrições devido a questões técnicas associadas com os equipamentos e tecnologias utilizadas [Leite, 2007].

Atualmente já são consideradas como amplamente disponíveis e com qualidade adequadas as aplicações de síntese e reconhecimento de voz, tanto pela disponibilidade de sistemas comerciais como pela existência de bibliotecas de desenvolvimento [Oliveira, et al, 2011]. Estas tecnologias de síntese e reconhecimento de voz são importantes pois atuam como apoio no caso de traduções de LIBRAS.

Entretanto a detecção de sinais depende de tecnologias específicas, também atualmente em estágio bastante amadurecido, sendo a seguir descritas e analisadas em maiores detalhes. Baseando-se na leitura de trabalhos na área, detectou-se a existência de duas abordagens gerais para reconhecimento da língua de sinais: a) abordagens baseadas em visão ou sinais; b) abordagens baseadas em luva (dataglove). Estas duas abordagens possuem vantagens e desvantagens quando comparadas entre si. Por exemplo, a necessidade do usuário vestir luvas para poder interagir com o sistema tradutor gera uma diminuição na usabilidade da interação humano-computador, porém apresenta um grande ganho quanto a precisão na captação de movimentos [Kuroda et al. 2004]. Já as características da abordagem baseada em visão permitem uma interação mais intuitiva com a máquina, pois não há a necessidade de vestir nenhum tipo de equipamento [Yang 2010]. Estes trabalhos apresentam a desvantagem, porém, da dificuldade de identificação das diferentes configurações de mão em diferentes orientações. Entretanto estes diversos aspectos estão sendo rapidamente superados, tanto com a integração de abordagens, como com a disponibilização de novos recursos tecnológicos.

No texto que segue serão apresentados resumos de diferentes técnicas e trabalhos relacionados à captação e interpretação da língua de sinais, que utilizam as 
duas abordagens apresentadas, com o intuito de proporcionar ao leitor o conjunto de informações necessário para uma avaliação desta evolução e para a identificação de possibilidades futuras.

\subsection{Abordagens baseadas em visão ou sinais}

Yang Quan em seu trabalho Chinese sign language recognition based on video sequence appearance modeling [Yang 2010] aborda uma técnica baseada em visão focando na identificação dos sinais que representam o alfabeto chinês. Para realizar a identificação de cada sinal, o autor desenvolveu um sistema que captura objetos em movimento, ignorando o cenário de fundo e destacando a mão do usuário. Com este passo identificado, é aplicado um filtro destacando as bordas que compõem a imagem da mão, tal como pode ser visto na Figura 3 a seguir.
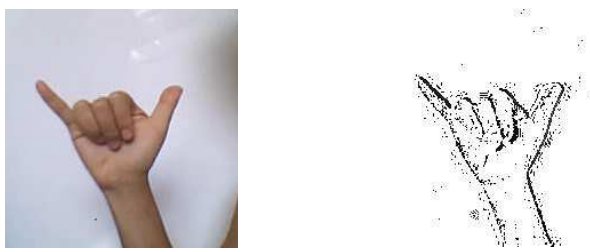

Figura 3 - Alfabeto manual [Yang 2010].

Através desta imagem resultante (observada na parte direita da Figura 3) é identificado o formato da mão, e por sua vez a letra que está sendo sinalizada. Os resultados descritos para este trabalho reportam acertos na faixa de $95 \%$ a 99\%, para as letras da Língua Chinesa de Sinais.

O Robust Person-Independent Visual Sign Language Recognition é um projeto que foca a tradução da British Sign Language (BSL) em uma abordagem baseada em visão e em duas formas: dependente e independente de usuário. Essa distinção é criada, pois sua taxa de sucesso pode variar amplamente (de $44,1 \%$ a $99,3 \%$ ) dependendo do usuário [Zieren e Kraiss 2005]. Para alcançar as maiores taxas de acerto no reconhecimento de cada sinal, é necessário que o futuro usuário do software treine o seu sistema, para que ele melhor se adapte as suas características na execução de cada sinal. Com o treinamento e o teste do mesmo em um ambiente controlado, o sistema dependente de usuário e ambiente controlado (como exemplificado na Figura 4a, abaixo) alcança uma média de acertos no reconhecimento dos sinais em cerca de 98,9\%, enquanto que em um ambiente real e independente de usuário (observado na Figura 4b) o sistema pode alcançar apenas $3,7 \%$.

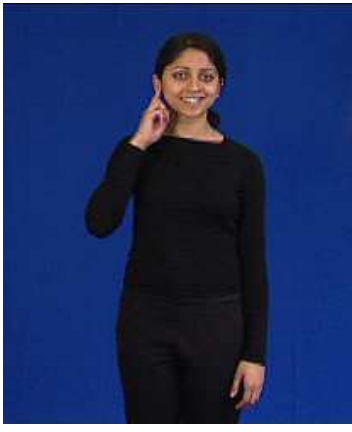

(a)

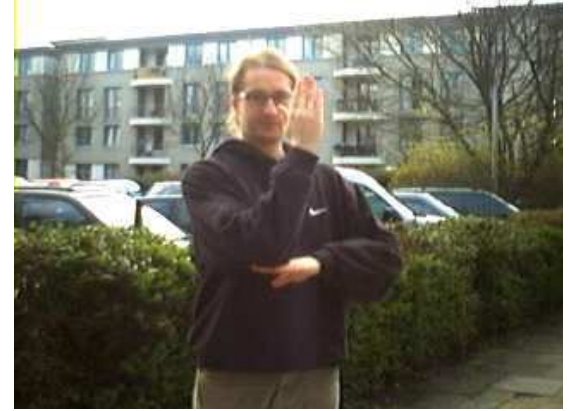

(b)

Figura 4 - Exemplos de treino/teste. a: Condição ideal. b: Condição do mundo real [Zieren e Kraiss 2005]. 
Projeto gerado pela Microsoft, o Digits busca criar um novo meio de interação Humano-Máquina, somando ao pulso do usuário um equipamento capaz de interpretar seus sinais manuais e movimentos de braço [Kim et al. 2012]. Isso ocorre por possuir canhão, laser e luz difusa todos em infravermelho e possuir um sensor capaz de detectar as três emissões separadamente, conforme ilustrado na Figura 5, além de usar suas características para identificar posição e ângulo dos dedos em relação ao pulso.

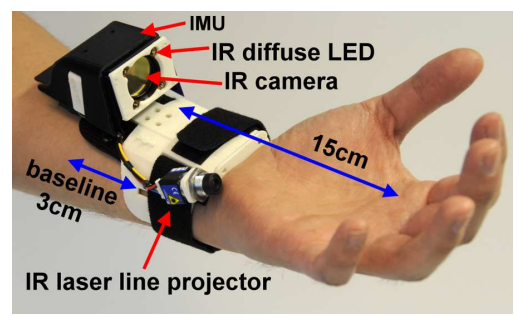

Figura 5 - Disposição dos elementos no hardware do Digits [Kim et al. 2012].

Com estas informações o software é capaz de reproduzir virtualmente a mão do usuário e interpretar seus gestos e sinais. $\mathrm{O}$ resultado desta interpretação pode resultar em diferentes interações como: mudar o canal da televisão, atender o telefone, abrir/fechar as cortinas da sala entre outras. Este projeto também não foi desenvolvido visando a comunicação em LIBRAS, por isso algumas limitações como os de movimento de pulso podem limitar e até prejudicar a comunicação entre os usuários.

\subsection{Abordagens baseadas em uso de luvas}

O projeto Sensor Libras visa a tradução interlínguas-intermodal da LIBRAS para oral-auditiva, o português brasileiro. $O$ foco deste trabalho encontra-se na datilologia dos sinais LIBRAS [Tavares, et al, 2010]. Para realizar a leitura da posição da mão, uma dataglove deve ser colocada na mão prioritária do deficiente (direita para destros, esquerda para canhotos). Uma segunda dataglove é colocada na outra mão, apenas para apresentar o retorno do módulo Feedback.

A luva colocada na mão prioritária possui um Sun Spot, hardware que capta sua movimentação e orientação nos eixos X Y Z em um ambiente, e os envia a uma base ligada a um computador, onde os cálculos são feitos, tal como descreve a Figura 6.
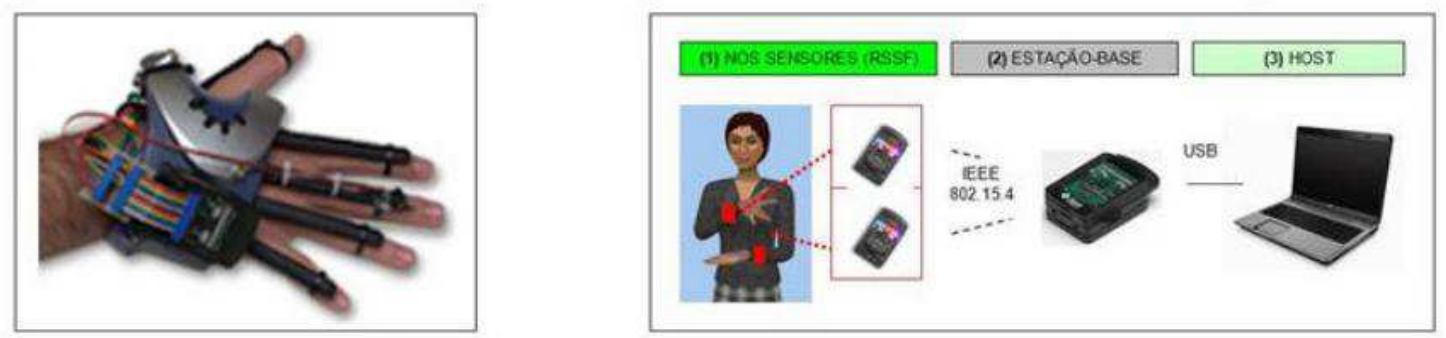

Figura 6 - Luva e arquitetura de comunicação SensorLibras [Tavares et al, 2010].

O software responsável pela realização da leitura, interpretação e tradução dos sinais LIBRAS para a língua portuguesa, está dividido em quatro módulos: (1) Reader, (2) Analyzer, (3) Parser e (4) Feedback. No módulo Reader, a dataglove realiza todas as leituras referentes ao posicionamento e movimentação da mão prioritária, gerando pontos nas coordenadas $\mathrm{X} \mathrm{Y}$ e $\mathrm{Z}$ a cada cinco milissegundos e as enviando a um computador host. No módulo Analyzer essas coordenadas são analisadas e interpretadas 
visando modelar matematicamente o sinal gesticulado. O módulo Parser é responsável por receber o modelo matemático construído, e buscar em sua base de dados, retornando o caractere correspondente. Caso o sinal seja encontrado, o Parser processa a saída deste para um arquivo XML e envia um sinal positivo ao módulo Feedback. Caso não encontre, o sinal retornado é negativo. O último módulo é o Feedback, ele é responsável por receber o sinal do módulo Parser, e acender na mão não prioritária, LED's que indicam na cor vermelha que o sinal não foi reconhecido, e na cor verde que a interpretação foi realizada com sucesso. Os autores indicam média na taxa de reconhecimento superior a $90 \%$.

Outra iniciativa nesta área, o projeto StringGove visa a construção de uma dataglove voltada especificamente para a tradução da língua de sinais [Kuroda et al. 2004]. Construída no Japão, a StrinGlove possui nove sensores de contato para captar o contato entre a ponta do polegar e diferentes pontos nos demais dedos. A Figura 7 ilustra esta descrição.

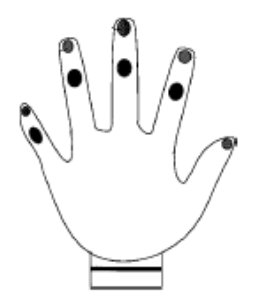

Figura 7 - Pontos de contato na luva [Kuroda et al. 2004].

Para calcular o ângulo de curvatura dos dedos da mão no projeto StringGlove, foram colocados componentes elétricos que permitem uma maior ou menor passagem de eletricidade conforme se aproximam de imãs, ou seja, quanto mais os dedos são dobrados, mais próximos estes componentes se aproximam de imãs e mais corrente elétrica é liberada. Essa corrente é identificada por um software que a interpreta e descobre exatamente a posição de cada dedo. Uma das principais vantagens deste sistema é o fato de que todos os componentes estão fixados a luva por meio de velcro (Figura 8), facilitando o seu processo de montagem/desmontagem e viabilizando o asseio do equipamento.

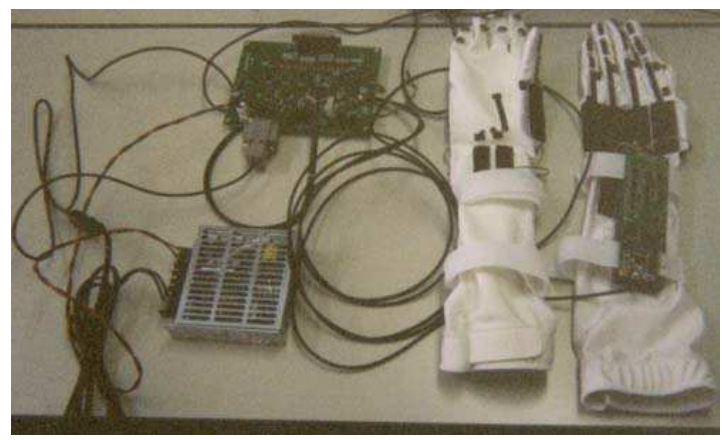

Figura 8 - Luvas montadas e prontas para o uso [Kuroda et al. 2004].

Em uma experiência interessante, Robert Y. Wang desenvolveu um sistema que utiliza o melhor das duas abordagens: a interação mais flexível através da câmera e a precisão das luvas [Wang e Popovié 2009]. Porém o diferencial no seu projeto é o uso 
de um padrão de cores distribuídas esquematicamente na luva, ao invés sensores, tal como observado nos outros projetos descritos. Através deste conjunto de cores o sistema verifica a posição de alguns pontos da mão, e os posiciona virtualmente representando e movimentando a mão virtual em tempo real de execução (Figura 9).
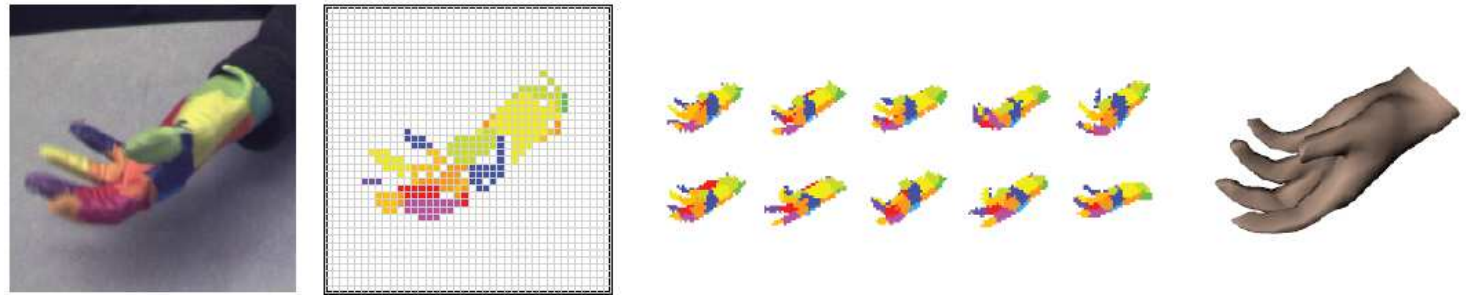

Figura 9 - Reconhecimento e interpretação da luva colorida [Wang e Popovié 2009].

O sistema foi desenvolvido para reconhecer, através da organização das cores percebidas, a disposição da mão do usuário. Segundo o autor, esta técnica apesar do maior custo computacional, trata problemas de oclusões, que são os pontos e locais da mão que não estão no campo de visão da câmera. Este projeto não foi desenvolvido especificamente para ser usado junto às línguas de sinais, mas sim para a interação humano máquina em diversos setores, o que demonstra o potencial do projeto.

\section{Análise dos trabalhos}

A tabela 1, abaixo, descreve as características dos trabalhos analisados, proporcionando uma comparação entre os mesmos, para diversos aspectos, descritos em suas colunas.

Tabela 1. Características dos trabalhos analisados.

\begin{tabular}{|c|c|c|c|c|c|}
\hline Projeto & $\begin{array}{l}\text { Reconhece } \\
\text { Datilologia }\end{array}$ & $\begin{array}{l}\text { Reconhece } \\
\text { língua de } \\
\text { sinais }\end{array}$ & $\begin{array}{l}\text { Projetado para } \\
\text { língua de sinais }\end{array}$ & $\begin{array}{l}\text { Taxa de acerto } \\
\text { maior que } 90 \%\end{array}$ & $\begin{array}{l}\text { É portátil e de } \\
\text { fácil manuseio }\end{array}$ \\
\hline $\begin{array}{l}\text { Chinese sign language recognition } \\
\text { based on video sequence appearance } \\
\text { modeling }\end{array}$ & Sim & Não & Sim & Sim & Sim \\
\hline SensorLibras & Sim & Não & Sim & Sim & Não \\
\hline $\begin{array}{l}\text { Robust Person-Independent Visual } \\
\text { Sign Language Recognition }\end{array}$ & Sim & Sim & Sim & Não & Sim \\
\hline StrinGlove & Sim & Sim & Sim & Sim & Não \\
\hline $\begin{array}{l}\text { Real-Time Hand-Tracking with a } \\
\text { Color Glove }\end{array}$ & Sim & Não & Não & Não avaliado & Sim \\
\hline Digits & Não & Não & Não & Não avaliado & Sim \\
\hline
\end{tabular}

A partir da verificação dos trabalhos mencionados, proporcionada pela tabela 1 , é possível identificar uma amostra do quão explorado é este segmento. Também resta evidenciado o interesse no tema, tendo em vista a grande quantidade de trabalhos desenvolvidos especificamente para esta finalidade.

Existem hoje diferentes abordagens para a resolução do mesmo problema, porém a precisão adequada na leitura dos movimentos está, até o momento, associada com o uso de algum equipamento junto das mãos do usuário. Para acompanhar fisicamente o movimento e configuração da mão na abordagem por luvas, observa-se um menor custo computacional, mas pode-se considerar alguma restrição na interação humano-computador. Mesmo assim, desta forma já existem soluções comerciais para esse problema, com ótimos resultados em termos de precisão.

Já para a abordagem por câmeras, onde a comunicação é mais natural, percebese a demanda por melhores recursos de hardware em termos de precisão, de modo a 
viabilizar esse desenvolvimento. Os trabalhos conhecidos realizam interpretações de movimentos simples e sem profundidade, desconsiderando parte da configuração de mão utilizada pelo usuário. Provavelmente por este motivo nenhum trabalho apresentou a interpretação de sinais semelhantes, mas com configurações de mão distintas, apenas a sinalização de sinais totalmente diferentes, onde com o apoio de uma rede neural é possível aprender/identificar cada sinal.

O fato da abordagem por câmeras, possível hoje, não conseguir realizar ainda tal discernimento, dificulta sua aplicação a contextos mais amplos, pois se assim ocorresse haveria restrição do poder de comunicação do usuário surdo. É possível, porém, antever a sua aplicabilidade para este contexto, com as novas tecnologias já descritas e em fase de disponibilização comercial, tais como, por exemplo, o Leap [LEAP 2012], que exibem um maior nível de precisão na detecção de profundidade e movimentos do usuário.

\section{Conclusões}

Neste trabalho foi apresentada uma contextualização do problema de identificação e tradução de sinais da LIBRAS, como forma de apoiar a integração dos usuários desta língua no contexto social e educacional. Observam-se diversas iniciativas, que atualmente apresentam restrições para identificação de sinais em contexto mais amplo. Entretanto, ao mesmo tempo estas diversas iniciativas atestam o nível de envolvimento da comunidade científica no assunto, o que pode trazer grandes benefícios aos alunos surdos no que se refere a sua inclusão nos processos rotineiros em escolas.

Além da detecção dos movimentos desenvolvidos por mão e dedos, se faz importante também a detecção das expressões faciais, que são úteis a comunicação em LIBRAS, por colocarem a entonação desejada a cada sinalização. Este ponto não é abordado por nenhum trabalho pesquisado, e traria um grande diferencial a um novo projeto a ser desenvolvido. Neste caso seria demandada a utilização de recursos baseados em câmera e processamento de imagens.

Os pontos levantados sugerem o futuro próximo com a viabilidade da detecção e interpretação da língua de sinais, onde uma comunicação mais dinâmica e aplicada a diversos contextos será possível. Em especial, promovendo a maior interação entre alunos em contexto escolar. Para tal, observam-se os avanços indicados nos trabalhos descritos, as tendências em melhorias em recursos de hardware, aliadas às possibilidades de integração, bem como a existência de recursos auxiliares, que permitem ampliar aspectos da interação multimodal, tal como na integração de aspectos de síntese e reconhecimento de voz.

\section{Referências}

Brito, L.F. (2011).A Gramática Da Libras. Disponível em <http://dc429.4shared.com/doc/LXAbBTnf/preview.html>. Acesso em: 15 out de 2012.

Coradine, L. C.; Albuquerque, F. C.; Brito, P. H. S.; Silva, R. L.; Silva, T. F. L. SistemaFalibras: Interpretação animada, em LIBRAS, de palavras e expressões em português. II Congresso Ibero-Americano de Informática na Educação Especial CIIEE. Córdoba-Argentina. 2002. 
10

Gesser, Audrei. Libras? Que língua é essa? São Paulo: Parábola Editorial, 2009.

Inforgestual. Língua Gestual Portuguesa, 2012. Disponível em <http://inforgestual.blogspot.com>. Acesso em: 27 out de 2012.

Kim, D., Hilliges, O., Izadi, S. and Butler, A. (2012). Digits: freehand 3D interactions anywhere using a wrist-worn gloveless sensor. Proceedings of the $25^{\text {th }}, \mathrm{p} .167-176$.

Kuroda, T., Tabata, Y. and Goto, A. (2004). Consumer price data-glove for sign language recognition. Proc. of 5th Intl Conf, p. 253-258.

Leap. Leap Motion, 2012. Disponível em < leapmotion.com>. Acesso em: 10 out de 2012.

Leite, M. D. Design da interação de interfaces educativaspara o ensino de matemática para crianças e jovens surdos. Dissertação de Mestrado. Universidade Federal de Pernambuco. AGOSTO/2007.

LIRA 2003, Lira, G. A. O Impacto da Tecnologia na Educação e Inclusão Social a Pessoa Portadorade Deficiência Auditiva: Tradutor Digital Português X Língua Brasileira de Sinais -Tlibras. BOLETIM TÉCNICO DO SENAC . Vol. 29, No 3, pp. 42-51, set./dez. 2003

Rafael Oliveira, Pedro Batista, Nelson Neto, Aldebaro Klautau. "Recursos para Desenvolvimento de Aplicativos com Suporte a Reconhecimento de Voz para Desktop e Sistemas Embarcados". Workshop de Software Livre (WSL), Porto Alegre, 2011

Tavares, João E. R. ; Barbosa, Jorge L. V. ; Leithardt, Valderi R. Q. . SensorLibras: Tradução Automática Libras-Português através da Computação Ubíqua. In: II Congresso Nacional de Pesquisa em Tradução e Interpretação de Língua de Sinais Brasileira, 2010, Florianópolis. Anais do II TILS, 2010. p. 1-7.

Wang, R. and PopoviÄł, J. (2009). Real-time hand-tracking with a color glove. ACM Transactions on Graphics (TOG), p. 1.

Yang, Q. (jun 2010). Chinese sign language recognition based on video sequence appearance modeling. 2010 5th IEEE Conference on Industrial Electronics and Applications, p. 1537-1542.

Zieren, J. and Kraiss, K. (2005). Robust person-independent visual sign language recognition. Pattern Recognition and Image Analysis,

Evandro Metz Flores, Sandro José Rigo, Uma ferramenta para apoio ao ensino da LIBRAS (Língua Brasileira de Sinais), RENOTE, v. 9, n. 3 (2011): Inclusão Digital e Educação Especial. 2011. 